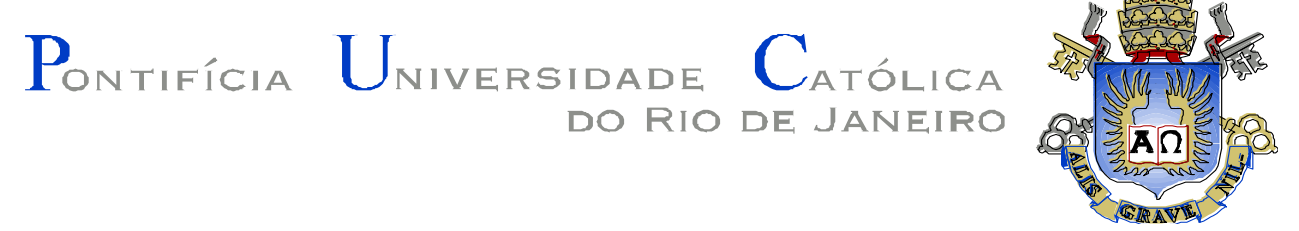

Luciana Brooking Teresa Dias

\title{
Memória de Crianças em Idade Escolar: Uma Perspectiva Neuropsicológica
}

Dissertação apresentada como requisito parcial para obtenção do grau de Mestre pelo Programa de PósGraduação em Psicologia Clínica do Departamento de Psicologia da PUC-Rio.

Orientador: Prof. Dr. Jesus Landeira-Fernandez

Rio de Janeiro

Março de 2011 
Pontificia Universidade C Católica

DO RIO DE JANEIRO

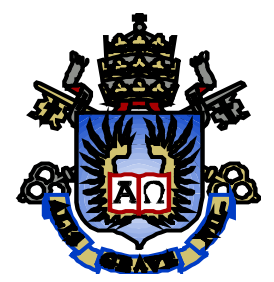

Luciana Brooking Teresa Dias

Memória de crianças em idade escolar: uma perspectiva neuropsicológica

Dissertação apresentada como requisito parcial para obtenção do grau de Mestre pelo Programa de Pós-Graduação em Psicologia Clínica do Departamento de Psicologia do Centro de Teologia e Ciências Humanas da PUC-Rio. Aprovada pela Comissão Examinadora abaixo assinada.

Prof. Jesus Landeira-Fernandez

Orientador

Departamento de Psicologia - PUC-Rio

Profa. Helenice Charchat-Fichman

Departamento de Psicologia - PUC-Rio

Profa. Rosinda Martins Oliveira Departamento de Psicometria - UFRJ

Profa. Denise Berruezo Portinari Coordenadora Setorial de Pós-Graduação

e Pesquisa do Centro de Teologia e Ciências Humanas - PUC-Rio

Rio de Janeiro, /2011 
Todos os direitos reservados. É proibida a reprodução total ou parcial do trabalho sem autorização do autor, do orientador e da universidade.

\section{Luciana Brooking Teresa Dias}

Graduou-se em Psicologia na UNESA (Universidade Estácio de Sá) em 2007. Formou-se em Psicoterapia CognitivoComportamental e Neuropsicologia. Tem atuado em clínica e pesquisas nessas áreas. Ministra aulas e palestras em áreas correlatas.

Ficha Catalográfica

Dias, Luciana Brooking Teresa

Memória de crianças em idade escolar : uma perspectiva neuropsicológica / Luciana Brooking Teresa

Dias ; orientador: Jesus Landeira-Fernandez. - 2011. 109 f. ; $30 \mathrm{~cm}$

Dissertação (mestrado)-Pontifícia Universidade

Católica do Rio de Janeiro, 2011.

Inclui bibliografia

1. Psicologia - Teses. 2. Sistemas de memória. 3. Memória explícita. 4. Neuropsicologia. 5. Criança. 6. Infância. 7. Desenvolvimento. I. Landeira- Fernandez, Jesus. II. Pontifícia Universidade Católica do Rio de Janeiro. Departamento de Psicologia. III. Título. 
Aos meus pais e avós, que me ensinaram os valores da memória 


\section{Agradecimentos}

A Deus, por tudo!!!

Aos meus pais pelo apoio, compreensão das ausências e investimento.

Ao meu marido Prof. Ercole Rubini pelos incentivos, investimento, orientação e paciência.

Aos amigos que sempre estiveram ao meu lado e me ajudaram com indicações.

Às escolas que permitiram a realização desse trabalho em suas instalações e a inserção desse extra em sua dinâmica diária.

Às crianças que participaram dessa pesquisa e possibilitaram dados e trocas riquíssimas. E aos seus responsáveis, por acreditar na importância desse trabalho ao autorizar a participação das crianças.

À CAPES e PUC-Rio pelos auxílios concedidos.

À professora Denise Morsch pelas palavras estimulantes e gentileza em ceder seu consultório para desenvolvimento da pesquisa.

Aos colegas do NNCE pelo apoio e força. Em especial à parceira Emmy Uehara pelas sugestões, trocas, organizações, e à colaboradora Gabriela Touche pela ajuda em todas as etapas da pesquisa.

Aos professores, responsáveis por minha formação nessa área, pelas contribuições, permitindo a construção desse caminho de pesquisa e de prática em neuropsicologia: Em especial: Regina Nogueira e à Banca, Rosinda Oliveira e Helenice Charchat-Fichman.

Ao meu orientador Landeira pela formação, contribuições e ensinamentos durante esse período. 


\section{Resumo}

Dias, Luciana Brooking Teresa; Landeira-Fernandez, Jesus (Orientador). Memória de Crianças em Idade Escolar: uma perspectiva neuropsicológica. Rio de Janeiro, 2011. 109p. Dissertação de Mestrado Departamento de Psicologia, Pontifícia Universidade Católica do Rio de Janeiro.

A memória se apresenta em sistemas distintos e interligados. Ela permite a constituição do sujeito e sua interação com o meio em que vive. Durante o desenvolvimento, mudanças biológicas e comportamentais vão ocorrendo, algumas vezes de forma rápida e outras, lentamente, respeitando a maturação neuronal, a interação social e a cultura em que vive. Nesse contexto, a emoção tem um papel modulador das funções cognitivas, fortalecendo ou enfraquecendo o armazenamento de uma informação, ou seja, influenciando a memória. Seu armazenamento pode ser sensorial, de curto e de longo prazo e ela pode se dividir em estágios (codificação, armazenamento e recuperação) e em tipos (explícita ou declarativa e implícita ou não declarativa). A memória explícita se subdivide em episódica e semântica, a implícita inclui os hábitos e habilidades, e a memória de curto prazo inclui a memória de trabalho. As áreas cerebrais envolvidas são o hipocampo, lobos frontal e temporal e a amígdala. Há distinção dos sistemas de memória durante o desenvolvimento: bebês reproduzem ações, reconhecem faces e eventos familiares e apresentam memória implícita (que não se altera muito ao longo do desenvolvimento); crianças pré-escolares apresentam uma memória mais sofisticada, organizando melhor as informações; e na fase escolar a memória já se encontra mais desenvolvida. $\mathrm{O}$ estudo mostrou que a memória semântica melhora gradualmente com a idade, acompanhando a ampliação de vocabulário; a memória episódica se desenvolve de forma mais pontual; e a memória de trabalho apresenta maturação mais tardia, acompanhando o desenvolvimento das funções executivas.

\section{Palavras-chave}

Sistemas de memória; memória explícita; neuropsicologia; criança; infância; desenvolvimento. 


\section{Abstract}

Dias, Luciana Brooking Teresa; Landeira-Fernandez, Jesus (Advisor). Memory in School-Aged Children: a neuropsychological perspective. Rio de Janeiro, 2011. 109p. MSc. Dissertation - Departamento de Psicologia, Pontifícia Universidade Católica do Rio de Janeiro.

The memory is divided into different systems and interconnected. It allows the creation of the subject and its interaction with the environment in which they live. During the development, behavioral and biological changes are occurring, sometimes quickly and others slowly, respecting the neuronal maturation, social interaction and culture in which they live. In this aspect, some skills are innate and others, acquired, learned. In this context, emotion plays a modulator of cognitive functions, strengthening or weakening the storage of information, ie, influencing memory. It can present divided into stages (sensory, short term and long term), in steps (encoding, storage and retrieval), and types (declarative or explicit and implicit or non-declarative). The explicit type is subdivided into episodic and semantic, the implicit include the habits and skills, and the short-term memory includes the working memory. The brain areas involved are the hippocampus, frontal and temporal lobes, and amygdale. in the formation of new memories and the recognition and consolidation during learning, and amygdala, allowing storage of the episodes that involve more emotion. There are distinctions in the memory systems during the development: babies reproduce actions, recognize faces and family events and have implicit memory (which does not change much throughout development), preschool children have a more sophisticated memory by organizing the information better; and during school memory is already more developed. The study showed that semantic memory improves gradually with age, following the expansion of vocabulary; episodic memory develops in a more timely, and working memory presents late maturation, following the development of executive functions.

\section{Keywords}

Memory systems; explicit memory; neuropsychology; child; childhood; development. 


\section{Sumário}

Apresentação

1. Introdução

1.1. Desenvolvimento Cognitivo

1.2. Aprendizagem

1.3. Emoção 15

1.4. Memória 17

1.4.1. Estágios de Memória 20

1.4.2. Sistemas de Memória $\quad 24$

1.4.3. Etapas da Memória $\quad 30$

1.5 Referências Bibliográficas 33

2. Neuropsicologia do desenvolvimento da memória: da pré-escola ao período escolar $\quad 41$

2.1. Resumo 41

2.2. Abstract 42

2.3. Introdução 43

2.4. Memórias: conceitos, tipos, componentes e correlatos Neurais $\quad 44$

2.5. Desenvolvimento da memória: da primeira à segunda infância, da fase pré-escolar à escolar 46

2.6. Considerações Finais 54

2.7. Referências $\quad 55$

3. Desenvolvimento da memória em crianças escolares de Classe Média

3.1. Resumo

3.2. Abstract

3.3. Introdução

3.4. Materiais e Métodos 
3.4.2. Procedimentos Experimentais $\quad 69$

3.4.3. Instrumentos $\quad 70$

3.4.3.1. "Parent And Children Interview For Psychiatric

Syndromes" (P-Chips)

70

3.4.3.2. Escala Multidimensional De Ansiedade Para Crianças

(Masc)-Multidimensional Anxiety Scale For Children (Masc) 70

3.4.3.3. Inventário de Depressão Infantil (CDI) 70

3.4.3.4. Teste de Desempenho Escolar (TDE) 71

3.4.3.5. Protocolo da Escala de Inteligência Weschler para Crianças (WISC)

3.4.3.6. Protocolo do Teste de Aprendizagem-Auditivo Verbal de Rey (RAVLT)

3.4.3.7. Protocolo do Teste de Fluência Verbal (Animal,

Roupa, Fruta)

72

3.4.3.8. Protocolo da Figura Complexa de Rey-Osterreith 72

3.4.4. Análise Estatística

3.5. Resultados

74

3.6. Discussão

76

3.7. Conclusão

79

3.8. Referências Bibliográficas

80

4. Considerações Finais

89

5. Referências Bibliográficas

6. Anexos

104

6.1. Termo de Consentimento Livre e Esclarecido

104

6.2. Gráficos 


\section{Apresentação}

A memória é uma função cognitiva que se apresenta na forma de sistemas distintos, porém, ligados uns aos outros. Através dela as pessoas conseguem se comunicar e organizar sua vida e seu self. Dessa forma, ela tem um papel vital para o indivíduo, pois a partir dela, ele se situa no espaço e no contexto em que está inserido. Os indivíduos vão guardando, ao longo da vida, experiências, os sentimentos ligados às experiências, os pensamentos relacionados a elas, e tudo isso vai construindo o sujeito, juntamente com as informações vivenciadas e perdidas.

Dentro desse contexto, uma das ciências que investiga a memória é a Neuropsicologia. Essa é uma disciplina que estuda a relação entre o cérebro e o comportamento humano, em sua atividade mental normal e/ou patológica. Da mesma forma, investiga as alterações cerebrais, seus efeitos sobre o comportamento e sua relação com o meio em que vive (Tabaquim, 2003). Seus estudos possibilitam um prognóstico, uma orientação de estratégias terapêuticas e elaboração de um programa de tratamento mais focado. Em seus objetivos, faz uso da avaliação neuropsicológica, ferramenta esta que envolve a utilização de entrevistas, observações e análise do contexto bem como testes e escalas. Dessa maneira, é possível estudarmos a percepção, atenção, memória, linguagem, pensamento, entre outras funções cognitivas (Luria, 1981).

No que se refere à memória e aprendizagem, a avaliação neuropsicológica permite investigar mais a fundo o processo e os sistemas de memória, possibilitando compreender seus déficits e prejuízos ao longo do aprendizado. Entretanto, estudar as funções cognitivas na infância apresenta algumas dificuldades, entre elas o fato do cérebro estar em constante desenvolvimento. Portanto, é importante que se considere não só as diferenças entre as faixas etárias, mas também, escolher as tarefas apropriadas para cada idade (Gulya, Rossi-George, Hartshorn, Vieira, \& Rovee-Collier, 2002).

Enquanto alguns sistemas não apresentam muita alteração ao longo da vida, outros mudam durante o desenvolvimento, seja aumentando ou reduzindo sua capacidade. Dentre as alterações que ocorrem ao longo do desenvolvimento, algumas delas estão presentes na fase de idade escolar. Por ser um assunto 
complexo, que ainda apresenta controvérsias sobre suas características, e por estar presente no cotidiano de todos, faz-se necessário seu aprofundamento.

Sendo assim, esta dissertação tem como objetivo investigar o desenvolvimento da memória, identificando suas características de funcionamento e suas principais mudanças. Contextualizando, assim, seus constructos na infância, dentro da visão da Neuropsicologia, especificamente fazendo uma análise em relação a gênero e idade.

A dissertação está dividida em quatro capítulos. O primeiro capítulo trará um aspecto geral sobre Desenvolvimento Cognitivo, Aprendizagem, Emoção e apresentará os Sistemas de Memória fundamentados na Psicologia Cognitiva. O objetivo desse capítulo é contextualizar o leitor no âmbito do desenvolvimento cognitivo e familiarizar nos conceitos de memória, a fim de facilitar a compreensão da leitura nos seguintes capítulos. O segundo capítulo será composto pelo artigo de revisão literária sobre o tema "Neuropsicologia do desenvolvimento da memória: da pré-escola ao período escolar". O objetivo desse estudo foi a contribuição para a organização do conhecimento sobre as bases mnemônicas, visando uma melhor compreensão das dificuldades que podem ocorrer na infância, assim como para a promoção de estratégias de estimulação de componentes mnemônicos nesta fase. Esse capítulo foi publicado na Revista Neuropsicologia Latinoamericana. Em seguida, o terceiro capítulo é constituído pelo ensaio teórico do artigo original intitulado "Desenvolvimento da memória em crianças escolares de classe média". Seu objetivo foi verificar a memória de crianças em idade escolar sob a perspectiva da Neuropsicologia. Finalmente, no quarto capítulo, haverá um fechamento da dissertação, de acordo com a literatura pesquisada e os dados obtidos nesse estudo. Além disso, serão ressaltadas as limitações e perspectivas de futuros estudos. No quinto capítulo serão apresentadas as referências bibliográficas da dissertação inteira, incluindo os capítulos de artigos a publicar.

Cabe ressaltar que os capítulos foram escritos considerando as normas da American Psychiatric Association (APA) em face às solicitações das Revistas de Psicologia a se submeter os artigos. 